\title{
SOME STATISTICAL PROPERTIES OF THE SOLUTIONS OF A SYSTEM OF TWO DIMENSIONAL STOCHASTIC FREDHOLM INTEGRAL EQUATIONS CONTAINS GAMMA PROCESSES
}

\author{
Mohammad Wahdan Muflih \\ Department of Mathematics, Faculty of Education (Ibn-Alhaitham), University of Baghdad, Iraq
}

Received 2012-12-20, Revised 2013-03-08; Accepted 2013-05-04

\begin{abstract}
In this study, we find the two solutions of a system of two dimensional stochastic Fredholm integral equations contains two gamma processes with different values of the two parameters (the first controls the rate of jump arrivals and the second inversely controls the jump size of this arrivals) in two cases and equal in the third that is to introduce two corresponding Probability Density Functions (PDF's) and to derive some statistical properties (auto-covariance and spectral density functions) depending upon the maximum variance of each p.d.f with respect to the three cases. To indicate which of the three cases gives a highest correlation, the correlation coefficients between any pair of p.d.f's related to every case are calculated. The solutions of the system of equations are found by the Adomian Decomposition Method (ADM), they are considered as a rapidly converging and geometrical infinite series. It is shown that, the highest correlation coefficient between any pair of p.d.f's is when the parameters of the two gamma processes are equal to one. This study is interesting as a main goal by combining two fields of mathematics, integral equations and probability theory that is by using the analytical solutions of a system of two dimensional stochastic Fredholm integral equations.
\end{abstract}

Keywords: System of Two Dimensional Stochastic Fredholm Integral Equations, Gamma Process, Adomin Decomposition Method

\section{INTRODUCTION}

Many researchers interested as a final goal either by studying the existence and uniqueness of the solution of one or more dimensional integral equations (Balachandran et al., 2005; Milton et al., 1972) or to find by using different methods of the modified quadrature the numerical solutions of this kind of equations on some definite closed interval to study a comparison between the numerical solutions and their exact solutions (Huda, 2007; AL-Sadany, 2008). While Vahidi and Mokhtari (2008) use the Adomian decomposition method to compare this method with the classical successive method for solving system of linear Fredholm integral equations and Biazar and Rangbar (2007) studied the comparison between Newton's method and Adomian decomposition method for solving special Fredholm integral equations.
For combining the integral equations as an important branch of mathematics with some statistical properties, our goal in this study is not only interesting in the solutions of the supposing a system of two-dimensional stochastic Fredholm integral equations but we concentrate ourselves in the derivation of some statistical properties of the solutions like probability density, covariance and spectral density functions depending upon the maximum variances of the probability density functions of the resulting stochastic two solutions of the system which found by the Adomian decomposition method.

\subsection{Preliminary}

The gamma process $\Gamma(\mathrm{w}, \gamma, \lambda, \mathrm{t})$ is a Lev'y process whose marginal distribution at the time $t>0$ is a gamma distribution with mean $\frac{\gamma t}{\lambda}$ and variance $\frac{\gamma t}{\lambda^{2}}$, i.e. Equation 1: 
$\Gamma(w, \gamma, \lambda, t)=\frac{(\lambda)^{\gamma t}}{\Gamma(\gamma t)} w^{\gamma t-1} e^{-\lambda w} \quad, w>0, t>0$

where, the parameter $\Gamma$ controls the rate of jump arrivals (shape parameter) and the scaling parameter $\lambda$ inversely controls the jump size (scale parameter) (Vladimir and Dimitrina, 2009). Moreover, the gamma process has the following properties:

- $\quad \Gamma(\mathrm{w}, \gamma, \lambda, \mathrm{t})=0$

- For any $0 \leq \mathrm{t} 1<\mathrm{t} 2<\ldots<\mathrm{tn}<\infty ; \Gamma\left(\mathrm{w}, \gamma, \lambda, \mathrm{t}_{2}\right)-\Gamma(\mathrm{w}$, $\left.\gamma, \lambda, \mathrm{t}_{1}\right) \ldots \Gamma\left(\mathrm{w}, \quad \gamma, \lambda, \mathrm{t}_{\mathrm{n}}\right)-\Gamma\left(\mathrm{w}, \quad \gamma, \lambda, \mathrm{t}_{\mathrm{n}-1}\right)$ are independent increments

- $\quad$ For any $\mathrm{s}<\mathrm{t} ; \Gamma(\mathrm{w}, \gamma, \lambda, \mathrm{t})-\Gamma(\mathrm{w}, \gamma, \lambda$, s) have the same distribution as $\Gamma(\mathrm{w}, \gamma, \lambda$, t-s $)$

Now, we consider the following two dimensional system of stochastic Fredholm integral Equation 2:

$$
\begin{aligned}
& \mathrm{u}_{1}(\mathrm{w}, \mathrm{t})=\Gamma_{\mathrm{i}}\left(\mathrm{w}, \gamma_{\mathrm{i}}, \lambda_{\mathrm{i}}, \mathrm{t}\right)+ \\
& \int_{0}^{\infty} \sum_{\mathrm{j}=1}^{2} \mathrm{k}_{\mathrm{ij}}(\mathrm{w}, \mathrm{s}, \mathrm{t}) \mathrm{u}_{\mathrm{j}}(\mathrm{s}, \mathrm{t}) \mathrm{ds}, \quad \mathrm{i}=1,2
\end{aligned}
$$

where, $-\mathrm{k}_{\mathrm{ij}}(\mathrm{w}, \mathrm{s}, \mathrm{t}), \mathrm{i}, \mathrm{j}=1,2$ are known stochastic kernels defined by $t>0, s \in S, S$ is a compact metric and having respectively the supposing formulas Equation 3:

$$
\mathrm{k}_{1 \mathrm{j}}(\mathrm{w}, \mathrm{s}, \mathrm{t})=\mathrm{se}^{-\mathrm{wt}}, \mathrm{k}_{2 \mathrm{j}}(\mathrm{w}, \mathrm{s}, \mathrm{t})=\mathrm{se}^{-\mathrm{wt} \mathrm{t}^{2}}, \quad \mathrm{j}=1,2
$$

$-u_{j}(s, t), j=1,2$ are scalar functions defined for $t>0, s>0$. By substituting (1), (3) into (2), we get Equation 4:

$$
\begin{gathered}
\mathrm{u}_{1}(\mathrm{w}, \mathrm{t})=\frac{\left(\lambda_{1}\right)^{\gamma_{1} \mathrm{t}}}{\Gamma\left(\gamma_{1} \mathrm{t}\right)} \mathrm{w}^{\gamma_{1} \mathrm{t}-1} \mathrm{e}^{-\lambda_{1} \mathrm{w}}+\int_{0}^{\infty} \mathrm{se}^{-\mathrm{wt}}\left[\mathrm{u}_{1}(\mathrm{~s}, \mathrm{t})+\mathrm{u}_{2}(\mathrm{~s}, \mathrm{t})\right] \mathrm{ds} \\
\mathrm{u}_{2}(\mathrm{w}, \mathrm{t})=\frac{\left(\lambda_{2}\right)^{\gamma_{2} \mathrm{t}}}{\Gamma\left(\gamma_{2} \mathrm{t}\right)} \mathrm{w}^{\gamma_{2} \mathrm{t}-2} \mathrm{e}^{-\lambda_{2} \mathrm{w}}+\int_{0}^{\infty} \mathrm{se}^{-\mathrm{wt} 2}\left[\mathrm{u}_{1}(\mathrm{~s}, \mathrm{t})+\mathrm{u}_{2}(\mathrm{~s}, \mathrm{t})\right] \mathrm{ds}
\end{gathered}
$$

\section{Remark (1)}

In this study three cases for the parameters $(\gamma, \lambda)$ should be considered:

- $\gamma_{1}>\lambda_{1}: \gamma_{1}=1, \lambda_{1}=0.5 ; \gamma_{2}>\lambda_{2} ; \gamma_{2}=1.5, \lambda_{2} 1$

- $\gamma_{1}<\lambda_{1}: \gamma_{1}=0.5, \lambda_{1}=1 ; \gamma_{2}<\lambda_{2}: \gamma_{2}=1, \lambda_{2}=1.5$

- $\gamma_{1}=\lambda_{1}=\gamma_{2}=\lambda_{2}=1$
Now, to find the stochastic solutions of the system (4), Adomian decomposition method should be used which briefly depends on the following steps Equation 5-7 (Vahidi and Mokhtari, 2008):

$$
\left.\begin{array}{c}
\mathrm{u}_{10}(\mathrm{w}, \mathrm{t})=\Gamma_{1}\left(\mathrm{w}, \gamma_{1}, \lambda_{1}, \mathrm{t}\right)=\frac{\left(\lambda_{1}\right)^{\gamma_{1} \mathrm{t}}}{\Gamma\left(\gamma_{1} \mathrm{t}\right)} \mathrm{w}^{\gamma_{1} \mathrm{t}-1} \mathrm{e}^{-\lambda_{1} \mathrm{w}} \\
\mathrm{u}_{20}(\mathrm{w}, \mathrm{t})=\Gamma_{2}\left(\mathrm{w}, \gamma_{2}, \lambda_{2}, \mathrm{t}\right)=\frac{\left(\lambda_{2}\right)^{\gamma_{2} \mathrm{t}}}{\Gamma\left(\gamma_{2} \mathrm{t}\right)} \mathrm{w}^{\gamma_{2} \mathrm{t}-2} \mathrm{e}^{-\lambda_{2} \mathrm{w}}
\end{array}\right\}
$$

$$
\left.\begin{array}{c}
\mathrm{u}_{10}(\mathrm{~s}, \mathrm{t})=\Gamma_{1}\left(\mathrm{w}, \gamma_{1}, \lambda_{1}, \mathrm{t}\right)=\frac{\left(\lambda_{1}\right)^{\gamma_{1} \mathrm{t}}}{\Gamma\left(\gamma_{1} \mathrm{t}\right)} \mathrm{s}^{\gamma_{1} \mathrm{t}-1} \mathrm{e}^{-\lambda_{1} \mathrm{~s}} \\
\mathrm{u}_{20}(\mathrm{~s}, \mathrm{t})=\Gamma_{2}\left(\mathrm{w}, \gamma_{2}, \lambda_{2}, \mathrm{t}\right)=\frac{\left(\lambda_{2}\right)^{\gamma_{2} \mathrm{t}}}{\Gamma\left(\gamma_{2} \mathrm{t}\right)} \mathrm{s}^{\gamma_{2} \mathrm{t}-2} \mathrm{e}^{-\lambda_{2} \mathrm{~s}}
\end{array}\right\}
$$

And:

$$
\left.\begin{array}{l}
\mathrm{u}_{1, \mathrm{~m}+1}(\mathrm{w}, \mathrm{t})=\int_{0}^{\infty} \mathrm{se}^{-\mathrm{wt}}\left[\mathrm{u}_{1 \mathrm{~m}}(\mathrm{~s}, \mathrm{t})+\mathrm{u}_{2 \mathrm{~m}}(\mathrm{~s}, \mathrm{t})\right] \mathrm{ds} \\
\mathrm{u}_{2, \mathrm{~m}+1}(\mathrm{w}, \mathrm{t})=\int_{0}^{\infty} \mathrm{se}^{-\mathrm{wt} 2}\left[\mathrm{u}_{1 \mathrm{~m}}(\mathrm{~s}, \mathrm{t})+\mathrm{u}_{2 \mathrm{~m}}(\mathrm{~s}, \mathrm{t})\right] \mathrm{ds}
\end{array}\right\}
$$

where, $\mathrm{m}=0,1,2 \ldots$

That is to get the following two stochastic solutions Equation 8:

$$
\left.\begin{array}{l}
\mathrm{u}_{1}(\mathrm{w}, \mathrm{t})=\mathrm{u}_{10}(\mathrm{w}, \mathrm{t})+\sum_{\mathrm{n}=1}^{\infty} \mathrm{u}_{1 \mathrm{n}}(\mathrm{w}, \mathrm{t}) \\
\mathrm{u}_{1}(\mathrm{w}, \mathrm{t})=\mathrm{u}_{20}(\mathrm{w}, \mathrm{t})+\sum_{\mathrm{n}=1}^{\infty} \mathrm{u}_{2 \mathrm{n}}(\mathrm{w}, \mathrm{t})
\end{array}\right\}
$$

So, for the first iteration $(\mathrm{m}=0)$, by $(6)$ and $(7)$ :

$$
\begin{aligned}
& \mathrm{u}_{1,1}(\mathrm{w}, \mathrm{t})=\int_{0}^{\infty} \mathrm{se}^{-\mathrm{wt}}\left[\frac{\left(\lambda_{1}\right)^{\gamma_{1} \mathrm{t}}}{\Gamma\left(\gamma_{1} \mathrm{t}\right)} \mathrm{s}^{\gamma_{1} \mathrm{t}-1} \mathrm{e}^{-\lambda_{1} \mathrm{~s}}+\frac{\left(\lambda_{2}\right)^{\gamma_{2} \mathrm{t}}}{\Gamma\left(\gamma_{2} \mathrm{t}\right)} \mathrm{s}^{\gamma_{2} \mathrm{t}-1} \mathrm{e}^{-\lambda_{2} \mathrm{~s}}\right] \mathrm{ds} \\
& \mathrm{u}_{2,1}(\mathrm{w}, \mathrm{t})=\int_{0}^{\infty} \mathrm{se}^{-\mathrm{wt} 2}\left[\frac{\left(\lambda_{1}\right)^{\gamma_{1} \mathrm{t}}}{\Gamma\left(\gamma_{1} \mathrm{t}\right)} \mathrm{s}^{\gamma_{1} \mathrm{t}-1} \mathrm{e}^{-\lambda_{1} \mathrm{~s}}+\frac{\left(\lambda_{2}\right)^{\gamma_{2} \mathrm{t}}}{\Gamma\left(\gamma_{2} \mathrm{t}\right)} \mathrm{s}^{\gamma_{2} \mathrm{t}-1} \mathrm{e}^{-\lambda_{2} \mathrm{~s}}\right] \mathrm{ds} \\
& \mathrm{u}_{1,1}(\mathrm{w}, \mathrm{t})=\mathrm{e}^{-\mathrm{wt}}\left[\frac{\left(\lambda_{1}\right)^{\gamma_{1} \mathrm{t}}}{\left(\lambda_{1}\right)^{\gamma_{1} \mathrm{t}+1}} \cdot \frac{\Gamma\left(\gamma_{1} \mathrm{t}+1\right)}{\Gamma\left(\gamma_{1} \mathrm{t}\right)}+\frac{\left(\lambda_{2}\right)^{\gamma_{2} \mathrm{t}}}{\left(\lambda_{2}\right)^{\gamma_{2} \mathrm{t}+1}} \cdot \frac{\Gamma\left(\gamma_{2} \mathrm{t}+1\right)}{\Gamma\left(\gamma_{2} \mathrm{t}\right)}\right] \\
& \left.\mathrm{u}_{2,1}(\mathrm{w}, \mathrm{t})=\mathrm{e}^{-\mathrm{wt} \mathrm{t}^{2}}\left[\frac{\left(\lambda_{1}\right)^{\gamma_{1} \mathrm{t}}}{\left(\lambda_{1}\right)^{\gamma_{1} \mathrm{t}+1}} \cdot \frac{\Gamma\left(\gamma_{1} \mathrm{t}+1\right)}{\Gamma\left(\gamma_{1} \mathrm{t}\right)}+\frac{\left(\lambda_{2}\right)^{\gamma_{2} \mathrm{t}}}{\left(\lambda_{2}\right)^{\gamma_{2} \mathrm{t}+1}} \cdot \frac{\Gamma\left(\gamma_{2} \mathrm{t}+1\right)}{\Gamma\left(\gamma_{2} \mathrm{t}\right)}\right]\right\}
\end{aligned}
$$

or Equation 9: 
$\left.\begin{array}{c}\mathrm{u}_{1,1}(\mathrm{w}, \mathrm{t})=\mathrm{te}^{-\mathrm{wt}}\left(\frac{\gamma_{1}}{\lambda_{1}}+\frac{\gamma_{2}}{\lambda_{2}}\right) \\ \mathrm{u}_{2,1}(\mathrm{w}, \mathrm{t})=\mathrm{te}^{-\mathrm{wt}^{2}}\left(\frac{\gamma_{1}}{\lambda_{1}}+\frac{\gamma_{2}}{\lambda_{2}}\right)\end{array}\right\}$

and for the second iteration $(\mathrm{m}=1)$, by $(7)$ and $(9)$ :

$$
\left.\begin{array}{l}
\mathrm{u}_{1,2}(\mathrm{w}, \mathrm{t})=\int_{0}^{\infty} \mathrm{se}^{-\mathrm{wt}}\left[\mathrm{te}^{-\mathrm{st}}\left(\frac{\gamma_{1}}{\lambda_{1}}+\frac{\gamma_{2}}{\lambda_{2}}\right)+\mathrm{te}^{-\mathrm{st}^{2}}\left(\frac{\gamma_{1}}{\lambda_{1}}+\frac{\gamma_{2}}{\lambda_{2}}\right)\right] \mathrm{ds} \\
\mathrm{u}_{2,2}(\mathrm{w}, \mathrm{t})=\int_{0}^{\infty} \mathrm{se}^{-\mathrm{wt}^{2}}\left[\mathrm{te}^{-\mathrm{st}}\left(\frac{\gamma_{1}}{\lambda_{1}}+\frac{\gamma_{2}}{\lambda_{2}}\right)+\mathrm{te}^{-\mathrm{st}^{2}}\left(\frac{\gamma_{1}}{\lambda_{1}}+\frac{\gamma_{2}}{\lambda_{2}}\right)\right] \mathrm{ds}
\end{array}\right\}
$$

or Equation 10:

$$
\left.\begin{array}{l}
\mathrm{u}_{1,2}(\mathrm{w}, \mathrm{t})=\left(\frac{\gamma_{1}}{\lambda_{1}}+\frac{\gamma_{2}}{\lambda_{2}}\right)\left[\mathrm{t}\left(\frac{\mathrm{t}^{2}+1}{\mathrm{t}^{4}}\right)\right] \mathrm{e}^{-\mathrm{wt}} \\
\mathrm{u}_{2,2}(\mathrm{w}, \mathrm{t})=\left(\frac{\gamma_{1}}{\lambda_{1}}+\frac{\gamma_{2}}{\lambda_{2}}\right)\left[\mathrm{t}\left(\frac{\mathrm{t}^{2}+1}{\mathrm{t}^{4}}\right)\right] \mathrm{e}^{-\mathrm{wt}}{ }^{2}
\end{array}\right\}
$$

and by repeating iterations for $\mathrm{m}=2,3, \ldots$ and adding (9), (10), we get:

$$
\left.\begin{array}{l}
\sum_{n=1}^{\infty} \mathrm{u}_{1 \mathrm{n}}(\mathrm{w}, \mathrm{t})=\left(\frac{1}{\lambda_{1}}+\frac{2}{\lambda_{2}}\right) \mathrm{e}^{-\mathrm{wt}} \sum_{\mathrm{k}=0}^{\infty} \mathrm{t}\left(\frac{\mathrm{t}^{2}+1}{\mathrm{t}^{4}}\right)^{\mathrm{k}} \\
\sum_{\mathrm{n}=1}^{\infty} \mathrm{u}_{2 \mathrm{n}}(\mathrm{w}, \mathrm{t})=\left(\frac{1}{\lambda_{1}}+\frac{2}{\lambda_{2}}\right) \mathrm{e}^{-\mathrm{wt}} \sum_{\mathrm{k}=0}^{\infty} \mathrm{t}\left(\frac{\mathrm{t}^{2}+1}{\mathrm{t}^{4}}\right)^{\mathrm{k}}
\end{array}\right\}
$$

where, $\sum_{\mathrm{k}=0}^{\infty} \mathrm{t}\left(\frac{\mathrm{t}^{2}+1}{\mathrm{t}^{4}}\right)^{\mathrm{k}}$ is a geometric series converges for $\mathrm{t} \geq 1.30$, hence Equation 11:

$$
\left.\begin{array}{l}
\sum_{n=1}^{\infty} \mathrm{u}_{1 \mathrm{n}}(\mathrm{w}, \mathrm{t})=\left(\frac{1}{\lambda_{1}}+\frac{2}{\lambda_{2}}\right)\left[\frac{\mathrm{t}^{5}}{\mathrm{t}^{4}-\left(\mathrm{t}^{2}+1\right)}\right] \mathrm{e}^{-\mathrm{wt}} \\
\sum_{\mathrm{n}=1}^{\infty} \mathrm{u}_{2 \mathrm{n}}(\mathrm{w}, \mathrm{t})=\left(\frac{1}{\lambda_{1}}+\frac{2}{\lambda_{2}}\right)\left[\frac{\mathrm{t}^{5}}{\mathrm{t}^{4}-\left(\mathrm{t}^{2}+1\right)}\right] \mathrm{e}^{-w t^{2}}
\end{array}\right\}
$$

Finally, by substituting (5) and (11) into (8) which represents the two stochastic solutions of (4), we get Equation 12:

$$
\begin{aligned}
& \mathrm{u}_{1}(\mathrm{w}, \mathrm{t})=\frac{\left(\lambda_{1}\right)^{\gamma_{1} \mathrm{t}}}{\Gamma\left(\gamma_{1} \mathrm{t}\right)} \mathrm{w}^{\gamma_{1} \mathrm{t}-1} \mathrm{e}^{-\lambda_{1} \mathrm{w}}\left(\frac{1}{\lambda_{1}}+\frac{2}{\lambda_{2}}\right)\left[\frac{\mathrm{t}^{5}}{\mathrm{t}^{4}-\left(\mathrm{t}^{2}+1\right)}\right] \mathrm{e}^{-\mathrm{wt}} \\
& \left.\mathrm{u}_{2}(\mathrm{w}, \mathrm{t})=\frac{\left(\lambda_{2}\right)^{\gamma_{2} \mathrm{t}}}{\Gamma\left(\gamma_{2} \mathrm{t}\right)} \mathrm{w}^{\gamma_{2} \mathrm{t}-2} \mathrm{e}^{-\lambda_{2} \mathrm{w}}\left(\frac{1}{\lambda_{1}}+\frac{2}{\lambda_{2}}\right)\left[\frac{\mathrm{t}^{5}}{\mathrm{t}^{4}-\left(\mathrm{t}^{2}+1\right)}\right] \mathrm{e}^{-\mathrm{wt}^{2}}\right\}
\end{aligned}
$$

where, $\mathrm{w}>0, \mathrm{t} \geq 1.30, \gamma_{\mathrm{i}}, \lambda_{\mathrm{i}}>0, \mathrm{i}=1,2$
Moreover, the stochastic solutions (12) can be considered as a stochastic solutions over the interval $0 \leq \mathrm{w} \leq 1$ for some $\mathrm{t} \geq 1.30$.

\subsection{Statistical Properties of the Stochastic Solutions}

In order to derive the statistical properties of the two stochastic solutions (12) over the interval $0 \leq \mathrm{w} \leq 1, \mathrm{t} \geq 1.30$, it must be that each of them is a probability density function (p.d.f.) of $(\mathrm{W}, \mathrm{t})$. So, we multiply them respectively by $\mathrm{A}$ and $\mathrm{B}$ and equate their integrals by one that is to find $\mathrm{A}$ and $\mathrm{B}$ which make each stochastic solution is a p.d.f., i.e., we write:

$$
\int_{0}^{1} \mathrm{Au}_{1}(\mathrm{w}, \mathrm{t}) \mathrm{dw}=1, \quad \int_{0}^{1} \mathrm{Bu}_{2}(\mathrm{w}, \mathrm{t}) \mathrm{dw}=1
$$

We start by the first integral:

$$
\begin{gathered}
\mathrm{A}\left[\frac{\left(\lambda_{1}\right)^{\gamma_{1} \mathrm{t}}}{\Gamma\left(\gamma_{1} \mathrm{t}\right)} \int_{0}^{1} \mathrm{w}^{\gamma_{1} \mathrm{t}-1} \mathrm{e}^{-\lambda_{1} \mathrm{w}} \mathrm{dw}+\left(\frac{\gamma 1}{\lambda_{1}}+\frac{\gamma_{2}}{\lambda_{2}}\right)\left(\frac{\mathrm{t}^{5}}{\mathrm{t}^{4}-\left(\mathrm{t}^{2}+1\right)}\right) \int_{0}^{1} \mathrm{e}^{-\mathrm{wt}} \mathrm{dw}\right]=1 \\
\mathrm{~A}\left[\frac{\left(\lambda_{1}\right)^{\gamma_{1} \mathrm{t}}}{\Gamma\left(\gamma_{1} \mathrm{t}\right)} \int_{0}^{1} \mathrm{w}^{\gamma_{1} \mathrm{t}-1}\left(1-\lambda_{1} \mathrm{w}+\frac{\left(\lambda_{1} \mathrm{w}\right)^{2}}{2 !}-\cdots\right) \mathrm{dw}+\right. \\
\left.\left(\frac{\gamma 1}{\lambda_{1}}+\frac{\gamma 2}{\lambda_{2}}\right)\left(\frac{\mathrm{t}^{5}}{\mathrm{t}^{4}-\left(\mathrm{t}^{2}+1\right)}\right) \int_{0}^{1} \mathrm{e}^{-\mathrm{wt}} \mathrm{dw}\right]=1 \\
\mathrm{~A}\left[\frac{\left(\lambda_{1}\right)^{\gamma_{1} \mathrm{t}}}{\Gamma\left(\gamma_{1} \mathrm{t}\right)} \int_{0}^{1} \sum_{\mathrm{n}=0}^{\infty}\left(-\lambda_{1}\right)^{\mathrm{n}} \frac{\mathrm{w}^{\gamma_{1} \mathrm{t}+\mathrm{n}-1}}{\mathrm{n} !}+\left(\frac{\gamma 1}{\lambda_{1}}+\frac{\gamma 2}{\lambda_{2}}\right)\left(\frac{\mathrm{t}^{5}}{\mathrm{t}^{4}-\left(\mathrm{t}^{2}+1\right)}\right) \int_{0}^{1} \mathrm{e}^{-\mathrm{wt}} \mathrm{dw}\right]=1 \\
\left.\mathrm{~A}\left[\frac{\left(\lambda_{1}\right)^{\gamma_{1} \mathrm{t}}}{\Gamma\left(\gamma_{1} \mathrm{t}\right)} \sum_{\mathrm{n}=0}^{\infty} \frac{\left(-\lambda_{1}\right)^{\mathrm{n}}}{\mathrm{n} !\left(\gamma_{1} \mathrm{t}+\mathrm{n}\right)}+\frac{\gamma 1}{\lambda_{1}}+\frac{\gamma 2}{\lambda_{2}}\right)\left(\frac{\mathrm{t}^{5}}{\mathrm{t}^{4}-\left(\mathrm{t}^{2}+1\right)}\right)\left(\frac{1-\mathrm{e}^{-\mathrm{t}}}{\mathrm{t}}\right)\right]=1
\end{gathered}
$$

So:

$$
A=\frac{1}{\frac{\left(\lambda_{1}\right)^{\gamma_{1} \mathrm{t}}}{\Gamma\left(\gamma_{1} \mathrm{t}\right)} \sum_{\mathrm{n}=0}^{\infty} \frac{\left(-\lambda_{1}\right)^{\mathrm{n}}}{\mathrm{n} !\left(\gamma_{1} \mathrm{t}+\mathrm{n}\right)}+\left(\frac{\gamma 1}{\lambda_{1}}+\frac{\gamma 2}{\lambda_{2}}\right)\left(\frac{\mathrm{t}^{4}\left(1-\mathrm{e}^{-\mathrm{t}}\right)}{\mathrm{t}^{4}-\left(\mathrm{t}^{2}+1\right)}\right)}
$$
of B:

While, the second integral gives the following value

$$
B=\frac{1}{\frac{\left(\lambda_{2}\right)^{\gamma_{2} \mathrm{t}}}{\Gamma\left(\gamma_{2} \mathrm{t}\right)} \sum_{\mathrm{n}=0}^{\infty} \frac{\left(-\lambda_{2}\right)^{\mathrm{n}}}{\mathrm{n} !\left(\gamma_{2} \mathrm{t}+\mathrm{n}\right)}+\left(\frac{\gamma 1}{\lambda_{1}}+\frac{\gamma 2}{\lambda_{2}}\right)\left(\frac{\mathrm{t}^{3}\left(1-\mathrm{e}^{-\mathrm{t}^{2}}\right)}{\mathrm{t}^{4}-\left(\mathrm{t}^{2}+1\right)}\right)}
$$


Hence, the two stochastic solutions (12) are probability density functions of $(\mathrm{W}, \mathrm{t})$ that is when:

$$
\begin{aligned}
& \phi_{1}(\mathrm{w}, \mathrm{t})= \\
& \frac{\frac{\left(\lambda_{1}\right)^{\gamma_{1} \mathrm{t}}}{\Gamma\left(\gamma_{1} \mathrm{t}\right)} \mathrm{w}^{\gamma_{1} \mathrm{t}-1} \mathrm{e}^{-\lambda_{1} \mathrm{w}}+\left(\frac{\gamma 1}{\lambda_{1}}+\frac{\gamma 2}{\lambda_{2}}\right)\left(\frac{\mathrm{t}^{5}}{\mathrm{t}^{4}-\left(\mathrm{t}^{2}+1\right)}\right) \mathrm{e}^{-\mathrm{wt}}}{\frac{\left(\lambda_{1}\right)^{\gamma_{1} \mathrm{t}}}{\Gamma\left(\gamma_{1} \mathrm{t}\right)} \sum_{\mathrm{n}=0}^{\infty} \frac{\left(-\lambda_{1}\right)^{\mathrm{n}}}{\mathrm{n} !\left(\gamma_{1} \mathrm{t}+\mathrm{n}\right)}+\left(\frac{\gamma 1}{\lambda_{1}}+\frac{\gamma 2}{\lambda_{2}}\right)\left(\frac{\mathrm{t}^{4}\left(1-\mathrm{e}^{-\mathrm{t}}\right)}{\mathrm{t}^{4}-\left(\mathrm{t}^{2}+1\right)}\right)}
\end{aligned}
$$

$$
\begin{aligned}
& \phi_{2}(\mathrm{w}, \mathrm{t})= \\
& \frac{\frac{\left(\lambda_{2}\right)^{\gamma_{2} \mathrm{t}}}{\Gamma\left(\gamma_{2} \mathrm{t}\right)} \mathrm{w}^{\gamma_{2} \mathrm{t}-2} \mathrm{e}^{-\lambda_{2} \mathrm{w}}+\left(\frac{\gamma 1}{\lambda_{1}}+\frac{\gamma 2}{\lambda_{2}}\right)\left(\frac{\mathrm{t}^{5}}{\mathrm{t}^{4}-\left(\mathrm{t}^{2}+1\right)}\right) \mathrm{e}^{-\mathrm{wt}{ }^{2}}}{\frac{\left(\lambda_{2}\right)^{\gamma 2 \mathrm{t}}}{\Gamma\left(\gamma_{2} \mathrm{t}\right)} \sum_{\mathrm{n}=0}^{\infty} \frac{\left(-\lambda_{2}\right)^{\mathrm{n}}}{\mathrm{n} !\left(\gamma_{2} \mathrm{t}+\mathrm{n}\right)}+\left(\frac{\gamma 1}{\lambda_{1}}+\frac{\gamma 2}{\lambda_{2}}\right)\left(\frac{\mathrm{t}^{3}\left(1-\mathrm{e}^{-\mathrm{t}^{2}}\right)}{\mathrm{t}^{4}-\left(\mathrm{t}^{2}+1\right)}\right)}
\end{aligned}
$$

For both Equation $13 \mathrm{a}$ and $0 \leq \mathrm{w} \leq 1, \mathrm{t} \geq 1.30, \gamma_{\mathrm{i}}>0, \lambda_{\mathrm{i}}>0, \mathrm{i}=1,2$.

\subsection{First and Second Means}

\section{First Mean:}

$$
\left.\begin{array}{l}
E_{\phi_{1}}(w, t)=\int_{0}^{1} w\left[\phi_{1}(w, t)\right] d w \\
E_{\phi_{2}}(w, t)=\int_{0}^{1} w\left[\phi_{2}(w, t)\right] d w
\end{array}\right\}
$$

We start by the first mean of $\varnothing_{1}(\mathrm{w}, \mathrm{t})$ :

$$
\begin{aligned}
& \mathrm{E}_{\phi_{1}}(\mathrm{w}, \mathrm{t})= \\
& \frac{\int_{0}^{1} \mathrm{w}\left[\frac{\left(\lambda_{1}\right)^{\gamma_{1} \mathrm{t}}}{\Gamma\left(\gamma_{1} \mathrm{t}\right)} \mathrm{w}^{\gamma_{1} \mathrm{t}-1} \mathrm{e}^{-\lambda_{1} \mathrm{w}}+\left(\frac{\gamma_{1}}{\lambda_{1}}+\frac{\gamma_{2}}{\lambda_{2}}\right)\left(\frac{\mathrm{t}^{5}}{\mathrm{t}^{4}-\left(\mathrm{t}^{2}+1\right)}\right) \mathrm{e}^{-\mathrm{wt}}\right] \mathrm{dw}}{\frac{\left(\lambda_{1}\right)^{\gamma_{1} \mathrm{t}}}{\Gamma\left(\gamma_{1} \mathrm{t}\right)} \sum_{\mathrm{n}=0}^{\infty} \frac{\left(-\lambda_{1}\right)^{\mathrm{n}}}{\mathrm{n} !\left(\gamma_{1} \mathrm{t}+\mathrm{n}\right)}+\left(\frac{\gamma_{1}}{\lambda_{1}}+\frac{\gamma_{2}}{\lambda_{2}}\right)\left(\frac{\mathrm{t}^{4}\left(1-\mathrm{e}^{-\mathrm{t}}\right)}{\mathrm{t}^{4}-\left(\mathrm{t}^{2}+1\right)}\right)}= \\
& \frac{\frac{\left(\lambda_{1}\right)^{\gamma_{1} \mathrm{t}}}{\Gamma\left(\gamma_{1} \mathrm{t}\right)} \int_{0}^{1}+\left(\frac{\gamma_{1}}{\lambda_{1}}+\frac{\gamma_{2}}{\lambda_{2}}\right)\left(\frac{\mathrm{t}^{5}}{\mathrm{t}^{4}-\left(\mathrm{t}^{2}+1\right)}\right) \int_{0}^{1} \mathrm{we}^{-\mathrm{wt}} \mathrm{dw}}{\frac{\left(\lambda_{1}\right)^{\gamma_{1} \mathrm{t}}}{\Gamma\left(\gamma_{1} \mathrm{t}\right)} \sum_{\mathrm{n}=0}^{\infty} \frac{\left(-\lambda_{1}\right)^{\mathrm{n}}}{\mathrm{n} !\left(\gamma_{1} \mathrm{t}+\mathrm{n}\right)}} \\
& +\left(\frac{\gamma_{1}}{\lambda_{1}}+\frac{\gamma_{2}}{\lambda_{2}}\right)\left(\frac{\mathrm{t}^{4}\left(1-\mathrm{e}^{-\mathrm{t}}\right)}{\mathrm{t}^{4}-\left(\mathrm{t}^{2}+1\right)}\right)
\end{aligned}
$$

or Equation 14:

$13 b$

$$
\begin{aligned}
& \mathrm{E}_{\phi_{1}}(\mathrm{w}, \mathrm{t})= \\
& \left.\frac{\left(\lambda_{1}\right)^{\gamma_{1} \mathrm{t}}}{\Gamma\left(\gamma_{1} \mathrm{t}\right)} \sum_{\mathrm{n}=0}^{\infty} \frac{\left(-\lambda_{1}\right)^{\mathrm{n}}}{\mathrm{n} !\left(\gamma_{1} \mathrm{t}+\mathrm{n}+1\right)}\right)+\left(\frac{\gamma_{1}}{\lambda_{1}}+\frac{\gamma_{2}}{\lambda_{2}}\right)\left(\frac{\mathrm{t}^{3}\left(1-(\mathrm{t}+1) \mathrm{e}^{-\mathrm{t}}\right)}{\mathrm{t}^{4}-\left(\mathrm{t}^{2}+1\right)}\right) \\
& \frac{\left(\lambda_{1}\right)^{\gamma_{1} \mathrm{t}}}{\Gamma\left(\gamma_{1} \mathrm{t}\right)} \sum_{\mathrm{n}=0}^{\infty} \frac{\left(-\lambda_{1}\right)^{\mathrm{n}}}{\mathrm{n} !\left(\gamma_{1} \mathrm{t}+\mathrm{n}\right)}+\left(\frac{\gamma_{1}}{\lambda_{1}}+\frac{\gamma_{2}}{\lambda_{2}}\right)\left(\frac{\mathrm{t}^{4}\left(1-\mathrm{e}^{-\mathrm{t}}\right)}{\mathrm{t}^{4}-\left(\mathrm{t}^{2}+1\right)}\right)
\end{aligned}
$$

While, the first mean of $\emptyset_{2}(w, t)$ :

$$
\begin{aligned}
& \mathrm{E}_{\phi_{2}}(\mathrm{w}, \mathrm{t})= \\
& \frac{\int_{0}^{1} \mathrm{w}\left[\frac{\left(\lambda_{2}\right)^{\gamma_{1} \mathrm{t}}}{\Gamma\left(\gamma_{2} \mathrm{t}\right)} \mathrm{w}^{\gamma_{2} \mathrm{t}-1} \mathrm{e}^{-\lambda_{2} \mathrm{w}}+\left(\frac{\gamma_{1}}{\lambda_{1}}+\frac{\gamma_{2}}{\lambda_{2}}\right)\left(\frac{\mathrm{t}^{5}}{\mathrm{t}^{4}-\left(\mathrm{t}^{2}+1\right)}\right) \mathrm{e}^{-\mathrm{wt}^{2}}\right] \mathrm{dw}}{\frac{\left(\lambda_{2}\right)^{\gamma_{2} \mathrm{t}}}{\Gamma\left(\gamma_{2} \mathrm{t}\right)} \sum_{\mathrm{n}=0}^{\infty} \frac{\left(-\lambda_{2}\right)^{\mathrm{n}}}{\mathrm{n} !\left(\gamma_{2} \mathrm{t}+\mathrm{n}\right)}+\left(\frac{\gamma_{1}}{\lambda_{1}}+\frac{\gamma_{2}}{\lambda_{2}}\right)\left(\frac{\mathrm{t}^{3}\left(1-\mathrm{e}^{-\mathrm{t}^{2}}\right)}{\mathrm{t}^{4}-\left(\mathrm{t}^{2}+1\right)}\right)}
\end{aligned}
$$

Or Equation 15:

$$
\begin{aligned}
& \mathrm{E}_{\phi_{2}}(\mathrm{w}, \mathrm{t})= \\
& \left.\frac{\left(\lambda_{1}\right)^{\gamma_{1} \mathrm{t}}}{\Gamma\left(\gamma_{1} \mathrm{t}\right)} \sum_{\mathrm{n}=0}^{\infty} \frac{\left(-\lambda_{1}\right)^{\mathrm{n}}}{\mathrm{n} !\left(\gamma_{1} \mathrm{t}+\mathrm{n}+1\right)}\right)+\left(\frac{\gamma_{1}}{\lambda_{1}}+\frac{\gamma_{2}}{\lambda_{2}}\right)\left(\frac{\mathrm{t}\left(1-\left(\mathrm{t}^{2}+1\right) \mathrm{e}^{-\mathrm{t}^{2}}\right)}{\mathrm{t}^{4}-\left(\mathrm{t}^{2}+1\right)}\right) \\
& \frac{\left(\lambda_{2}\right)^{\gamma_{2} \mathrm{t}}}{\Gamma\left(\gamma_{2} \mathrm{t}\right)} \sum_{\mathrm{n}=0}^{\infty} \frac{\left(-\lambda_{2}\right)^{\mathrm{n}}}{\mathrm{n} !\left(\gamma_{2} \mathrm{t}+\mathrm{n}\right)}+\left(\frac{\gamma_{1}}{\lambda_{1}}+\frac{\gamma_{2}}{\lambda_{2}}\right)\left(\frac{\mathrm{t}^{3}\left(1-\mathrm{e}^{-\mathrm{t}^{2}}\right)}{\mathrm{t}^{4}-\left(\mathrm{t}^{2}+1\right)}\right)
\end{aligned}
$$

\section{Second Mean:}

$$
\left.\begin{array}{l}
E_{\phi_{1}}\left(w^{2}, t\right)=\int_{0}^{1} w^{2}\left[\phi_{1}(w, t)\right] d w \\
E_{\phi_{2}}\left(w^{2}, t\right)=\int_{0}^{1} w^{2}\left[\phi_{2}(w, t)\right] d w
\end{array}\right\}
$$

It is easy to derive the second means of $\emptyset_{2}(w, t)$, $\emptyset_{2}(\mathrm{w}, \mathrm{t})$ as pervious, they will be Equation 16:

$\mathrm{E}_{\phi_{1}}\left(\mathrm{w}^{2}, \mathrm{t}\right)=$

$$
\frac{\left.\frac{\left(\lambda_{1}\right)^{\gamma_{1} \mathrm{t}}}{\Gamma\left(\gamma_{1} \mathrm{t}\right)} \sum_{\mathrm{n}=0}^{\infty} \frac{\left(-\lambda_{1}\right)^{\mathrm{n}}}{\mathrm{n} !\left(\gamma_{1} \mathrm{t}+\mathrm{n}+2\right)}\right)+\left(\frac{\gamma_{1}}{\lambda_{1}}+\frac{\gamma_{2}}{\lambda_{2}}\right)\left(\frac{\mathrm{t}^{2}\left(2-\left(\mathrm{t}^{2}+2 \mathrm{t}+2\right) \mathrm{e}^{-\mathrm{t}}\right)}{\mathrm{t}^{4}-\left(\mathrm{t}^{2}+1\right)}\right)}{\frac{\left(\lambda_{1}\right)^{\gamma_{1}}}{\Gamma\left(\gamma_{1} \mathrm{t}\right)} \sum_{\mathrm{n}=0}^{\infty} \frac{\left(-\lambda_{1}\right)^{\mathrm{n}}}{\mathrm{n} !\left(\gamma_{1} \mathrm{t}+\mathrm{n}\right)}+\left(\frac{\gamma_{1}}{\lambda_{1}}+\frac{\gamma_{2}}{\lambda_{2}}\right)\left(\frac{\mathrm{t}^{4}\left(1-\mathrm{e}^{-\mathrm{t}}\right)}{\mathrm{t}^{4}-\left(\mathrm{t}^{2}+1\right)}\right)}
$$

While Equation 17:

$$
\begin{aligned}
& \mathrm{E}_{\phi_{2}}\left(\mathrm{w}^{2}, \mathrm{t}\right)= \\
& \left.\frac{\left(\lambda_{2}\right)^{\gamma_{2} \mathrm{t}}}{\Gamma\left(\gamma_{2} \mathrm{t}\right)} \sum_{\mathrm{n}=0}^{\infty} \frac{\left(-\lambda_{2}\right)^{\mathrm{n}}}{\mathrm{n}\left(\gamma_{2} \mathrm{t}+\mathrm{n}+2\right)}\right)+\left(\frac{\gamma_{1}}{\lambda_{1}}+\frac{\gamma_{2}}{\lambda_{2}}\right)\left(\frac{\mathrm{t}\left(2-\left(\mathrm{t}^{4}+2 \mathrm{t}^{2}+2\right) \mathrm{e}^{-t^{2}}\right)}{\mathrm{t}^{4}-\left(\mathrm{t}^{2}+1\right)}\right) \\
& \frac{\left(\lambda_{2}\right)^{\gamma_{2} \mathrm{t}}}{\Gamma\left(\gamma_{2} \mathrm{t}\right)} \sum_{\mathrm{n}=0}^{\infty} \frac{\left(-\lambda_{2}\right)^{\mathrm{n}}}{\mathrm{n} !\left(\gamma_{2} \mathrm{t}+\mathrm{n}\right)}+\left(\frac{\gamma_{1}}{\lambda_{1}}+\frac{\gamma_{2}}{\lambda_{2}}\right)\left(\frac{\mathrm{t}^{3}\left(1-\mathrm{e}^{-\mathrm{t}^{2}}\right)}{\mathrm{t}^{4}-\left(\mathrm{t}^{2}+1\right)}\right)
\end{aligned}
$$




\subsection{Variances and Maximum Variances}

Calculations of the variances of the probability density functions (p.d.f's) (13.a), (13.b) by the formula $\operatorname{var}_{\varnothing}(\mathrm{w}, \mathrm{t})=\mathrm{E}_{\emptyset}\left(\mathrm{w}^{2}, \mathrm{t}\right)-\left[\mathrm{E}_{\varnothing}(\mathrm{w}, \mathrm{t})\right]^{2}$ for the three cases (Remark (2.1)) when $t \geq 1.30$ are tabulated in Table $\mathbf{1}$ and 2. It is noted that, the variances in both tables are slowly decreasing to tends to zero when $\mathrm{t}$ is relatively large.

Whereas the maximum variances obtained from both presiding tables are presented respectively in the first, second and third rows in Table 3.

Furthermore, the probability density functions (13a) and (13b) with respect to the three cases Remark (2.1) can be rewritten respectively as follows:

- $\gamma_{1}>\lambda_{1}: \gamma_{1}=1, \lambda_{1}=0.5 ; \gamma_{2}>\lambda_{2}: \gamma_{2}=1.5, \lambda_{2}=1$ Equation $18 \mathrm{a}$

$\phi_{1}(w, 1.30)=0.01028 \mathrm{w}^{0.30} \mathrm{e}^{-0.5 \mathrm{w}}+1.77618 \mathrm{e}^{-1.30 \mathrm{w}}$

where, $\mathrm{t}=1.30,0 \leq \mathrm{w} \leq 1$ Equation $18 \mathrm{~b}$ :

$\phi_{2}(\mathrm{w}, 1.95)=0.19705 \mathrm{w}^{1.925} \mathrm{e}^{-\mathrm{w}}+0.376232 \mathrm{e}^{-3.8025 \mathrm{w}}$

where, $\mathrm{t}=1.95,0 \leq \mathrm{w} \leq 1$ Equation $18 \mathrm{c}$ :

- $\gamma_{1}<\lambda_{1}: \gamma_{1}=0.5, \lambda_{1}=1 ; \gamma_{2}<\lambda_{2}: \gamma_{2}=1, \lambda_{2}=1.5$

$\phi_{1}(\mathrm{w}, 1.30)=0.02799 \mathrm{w}^{-0.35} \mathrm{e}^{-\mathrm{w}}+1.73276 \mathrm{e}^{-1.30 \mathrm{w}}$

where, $\mathrm{t}=1.30,0 \leq \mathrm{w} \leq 1$ Equation $18 \mathrm{~d}$ :

$\phi_{2}(w, 2.0)=0.59100 \mathrm{we}^{-1.5 \mathrm{w}}+0.42030 \mathrm{e}^{-4 \mathrm{w}}$

where, $\mathrm{t}=2.0,0 \leq \mathrm{w} \leq 1$

- $\gamma_{1}=\lambda_{1}=\gamma_{2}=\lambda_{2}=1$ Equation 18e:

$\phi_{1}(\mathrm{w}, 1.30)=0.04350 \mathrm{w}^{-0.30} \mathrm{e}^{-\mathrm{w}}+1.74570 \mathrm{e}^{-1.30}$

where, $\mathrm{t}=1.30,0 \leq \mathrm{w} \leq 1$ Equation 18f:

$\phi_{2}(w, 2.0)=0.56080 \mathrm{we}^{-1.5 \mathrm{w}}+1.81280 \mathrm{e}^{-4 \mathrm{w}}$

where, $\mathrm{t}=2.0,0 \leq \mathrm{w} \leq 1$.

Figure 1-3 represent the graphs of three pairs of p.d.f's $\emptyset_{1}(\mathrm{w}, \mathrm{t}), \emptyset_{2}(\mathrm{w}, \mathrm{t})$.

\subsection{Autcovariance Function}

For any $\mathrm{s}>\mathrm{t}, \mathrm{s}-\mathrm{t}=\tau$, the autocovariance function $\mathrm{h}(\tau)$ of the independent p.d.f functions $\phi(w, t)$ and $\phi(w, t+\tau)$ is an even function depends on the difference $|\tau|=\mid$ s-t $\mid=$ $|\mathrm{t}-\mathrm{s}|$ and can be found as follows (Basu, 2003):

$$
\begin{aligned}
h(t)= & E_{\phi}((w, t)(w, t+t))-E_{\phi}(w, t) E_{\phi}(w, t+t) \\
= & E_{\phi}\left(w^{2}, t\right)+E_{\phi}((w, t)(w, t+t))-E_{\phi}\left(w^{2}, t\right)- \\
& E_{\phi}(w, t) E_{\phi}(w, t+t) \\
= & E_{\phi}\left(w^{2}, t\right)+E_{\phi}(w, t) E_{\phi}(w, t+t)-E_{\phi}\left(w^{2}, t\right)- \\
& E_{\phi}(w, t) E_{\phi}(w, t+t) \\
= & E_{\phi}\left(w^{2}, t\right)+E_{\phi}(w, t)\left(E_{\phi}(w, t+t)-E_{\phi}(w, t)\right)- \\
& E_{\phi}(w, t) E_{\phi}(w, t+t) \\
= & E_{\phi}\left(w^{2}, t\right)+E_{\phi}(w, t) E_{\phi}(w, t+t)-\left(E_{\phi}(w, t)\right)^{2}- \\
& E_{\phi}(w, t) E_{\phi}(w, t+t)
\end{aligned}
$$

Or:

$$
h(t)=E_{\phi}\left(w^{2}, t\right)-\left(E_{\phi}(w, t)\right)^{2}=\operatorname{var}_{\phi}(w, t)
$$

where, $\tau>0,0 \leq w \leq 1$.

So, by Table 3 and Equation (19) either when $\phi_{1}(w$, $\mathrm{t}), \mathrm{t}=1.30$ or $\phi_{2}(\mathrm{w}, \mathrm{t}), \mathrm{t}=1.95,2.0$, the auto-covariance functions for them with respect to the three cases (Remark 2.1) are presented in Table 4.

\subsection{Spectral Density Functions}

The spectral density functions (s.d,f) of $\phi(w, t)$ is an even function represents the average power in the p.d.f $\phi(w, t)$ at the angular frequency $0<\theta \leq 2 n \pi, n \in I^{+}$and can be found by Khinchin's formula as follows (Emanuel, 1962):

$$
\begin{aligned}
\mathrm{f}(\phi, \theta) & =\frac{1}{2 \pi} \int_{-\infty}^{\infty} \mathrm{h}(\tau) \mathrm{e}^{-\mathrm{i} \theta} \mathrm{d} \tau \mathrm{a} \\
& =\frac{\mathrm{h}(\tau)}{2 \pi} \int_{-\infty}^{\infty}(\cos \theta \tau-\sin \theta \tau) \mathrm{d} \tau
\end{aligned}
$$

and for $\tau=\mathrm{s}-\mathrm{t}>0$ :

$$
\left.f(\phi, \theta)=\frac{h(\tau)}{\pi} \int_{0}^{s-t} \cos \theta \tau-\sin \theta \tau\right) d \tau
$$

Or Equation 20:

$f(\phi, \theta)=\frac{h(\tau)}{\pi} \cdot \frac{\sin ((s-t) \theta)}{\theta}, 0<\theta \leq 2 n \pi, n \in I^{+}$

JMSS 
Table 1. Variances of $\phi_{1}(\mathrm{w}, \mathrm{t})$

\begin{tabular}{llll}
\hline & $\gamma_{1}>\lambda_{1}: \gamma_{1}=1, \lambda_{1}=0.5$ & $\gamma_{1}<\lambda_{1}: \gamma_{1}=0.5, \lambda_{1}=1$ & \\
$\mathrm{t}$ & $\gamma_{2}>\lambda_{2}: \gamma_{2}=1.5, \lambda_{2}=1$ & $\gamma_{2}<\lambda_{2}: \gamma_{2}=1, \lambda_{2}=1.5$ & $\gamma_{1}=\lambda_{1}=\gamma_{2}=\lambda_{2} 1$ \\
\hline 1.30 & 0.07683 & 0.07696 & 0.07689 \\
1.40 & 0.07620 & 0.07633 & 0.07658 \\
1.50 & 0.07549 & 0.07551 & 0.07633 \\
1.60 & 0.07468 & 0.07472 & 0.07607 \\
1.70 & 0.07377 & 0.07402 & 0.07577 \\
1.80 & 0.07276 & 0.07340 & 0.07539 \\
1.90 & 0.07166 & 0.07285 & 0.07491 \\
2.00 & 0.07049 & 0.07236 & 0.07430 \\
2.10 & 0.06924 & 0.07193 & 0.07356 \\
2.20 & 0.06793 & 0.07154 & 0.07269 \\
2.30 & 0.06658 & 0.07117 & 0.07169 \\
2.40 & 0.06520 & 0.07082 & 0.07056 \\
2.50 & 0.06379 & 0.07048 & 0.06931 \\
$\cdot$ & $\cdot$ & $\cdot$ &. \\
. & $\cdot$ & $\cdot$ &. \\
1000 & - &. & 0.000001 \\
\hline
\end{tabular}

Table 2. Variances of $\phi_{2}(\mathrm{w}, \mathrm{t})$

\begin{tabular}{llll}
\hline & $\gamma_{1}>\lambda_{1}: \gamma_{1}=1, \lambda_{1}=0.5$ & $\gamma_{1}<\lambda_{1}: \gamma_{1}=0.5, \lambda_{1}=1$ & $\gamma_{1}=\lambda_{1}=\gamma_{2}=\lambda_{2} 1$ \\
$\mathrm{t}$ & $\gamma_{2}>\lambda_{2}: \gamma_{2}=1.5, \lambda_{2}=1$ & $\gamma_{2}<\lambda_{2}: \gamma_{2}=1, \lambda_{2}=1.5$ & 0.21209 \\
1.30 & 0.21423 & 0.21009 & 0.22609 \\
1.35 & 0.23192 & 0.22072 & 0.25095 \\
1.45 & 0.26342 & 0.23964 & 0.27157 \\
1.55 & 0.28935 & 0.25529 & 0.28780 \\
1.65 & 0.30928 & 0.26752 & 0.29957 \\
1.75 & 0.32308 & 0.27631 & 0.30698 \\
1.85 & 0.33089 & 0.28184 & 0.31033 \\
1.95 & 0.33313 & 0.28438 & 0.31061 \\
2.00 & 0.32968 & 0.28466 & 0.30665 \\
2.15 & 0.32376 & 0.28218 & 0.30074 \\
2.25 & 0.31387 & 0.27835 & 0.29286 \\
2.35 & 0.30167 & 0.27329 & 0.28357 \\
2.45 & 0.28798 & 0.26738 & 0.27331 \\
2.55 & 0.27349 & 0.26092 &. \\
$\cdot$ & $\cdot$ & $\cdot$ &. \\
$\cdot$ & $\cdot$ & $\cdot$ & 0.000002 \\
1000 & 0.000002 &. & 0.000002 \\
\hline
\end{tabular}

Table 3. Presents the maximum variances from both Table 1 and 2

\begin{tabular}{lllll}
\hline & & $\gamma_{1}<\lambda_{1}: \gamma_{1}=0.5, \lambda_{1}=1$ & \\
p.d.f. & $\mathrm{t}$ & $\gamma_{2}>\lambda_{1}: \gamma_{1}=1, \gamma_{2}=1.5, \lambda_{2}=1$ & $\gamma_{2}<\lambda_{2}: \gamma_{2}=1, \lambda_{2}=1.5$ & $\gamma_{1}=\lambda_{1}=\gamma_{2}=\lambda_{2} 1$ \\
\hline$\phi_{1}(\mathrm{w}, \mathrm{t})$ & 1.30 & 0.07683 & 0.07696 & 0.07689 \\
$\phi_{2}(\mathrm{w}, \mathrm{t})$ & 1.95 & 0.33313 & - & - \\
& 2.0 & - & 0.28466 & 0.31061 \\
\hline
\end{tabular}

Table 4. Presents the Auto-covariance Functions of $\phi(w, t)$ and $\phi(w, t+\tau)$

\begin{tabular}{llll}
\hline & $\gamma_{1}>\lambda_{1}: \gamma_{1}=1, \lambda_{1}=0.5$ & $\gamma_{1}>\lambda_{1}: \gamma_{1}=0.5, \lambda_{1}=1$ & \\
$\mathrm{t}$ & $\gamma_{2}>\lambda_{2}: \gamma_{2}=1.5, \lambda_{2}=1$ & $\gamma_{2}>\lambda_{2}: \gamma_{2}=1, \lambda_{2}=1.5$ & $\gamma_{1}=\lambda_{1}=\gamma_{2}=\lambda_{2}=1$ \\
\hline 1.30 & 0.07683 & 0.07696 & 0.07689 \\
1.95 & 0.33313 & ---- & -- \\
2.00 & ---- & 0.28466 & 0.31061 \\
\hline
\end{tabular}


Mohammad Wahdan Muflih / Journal of Mathematics and Statistics 9 (2): 91-101, 2013

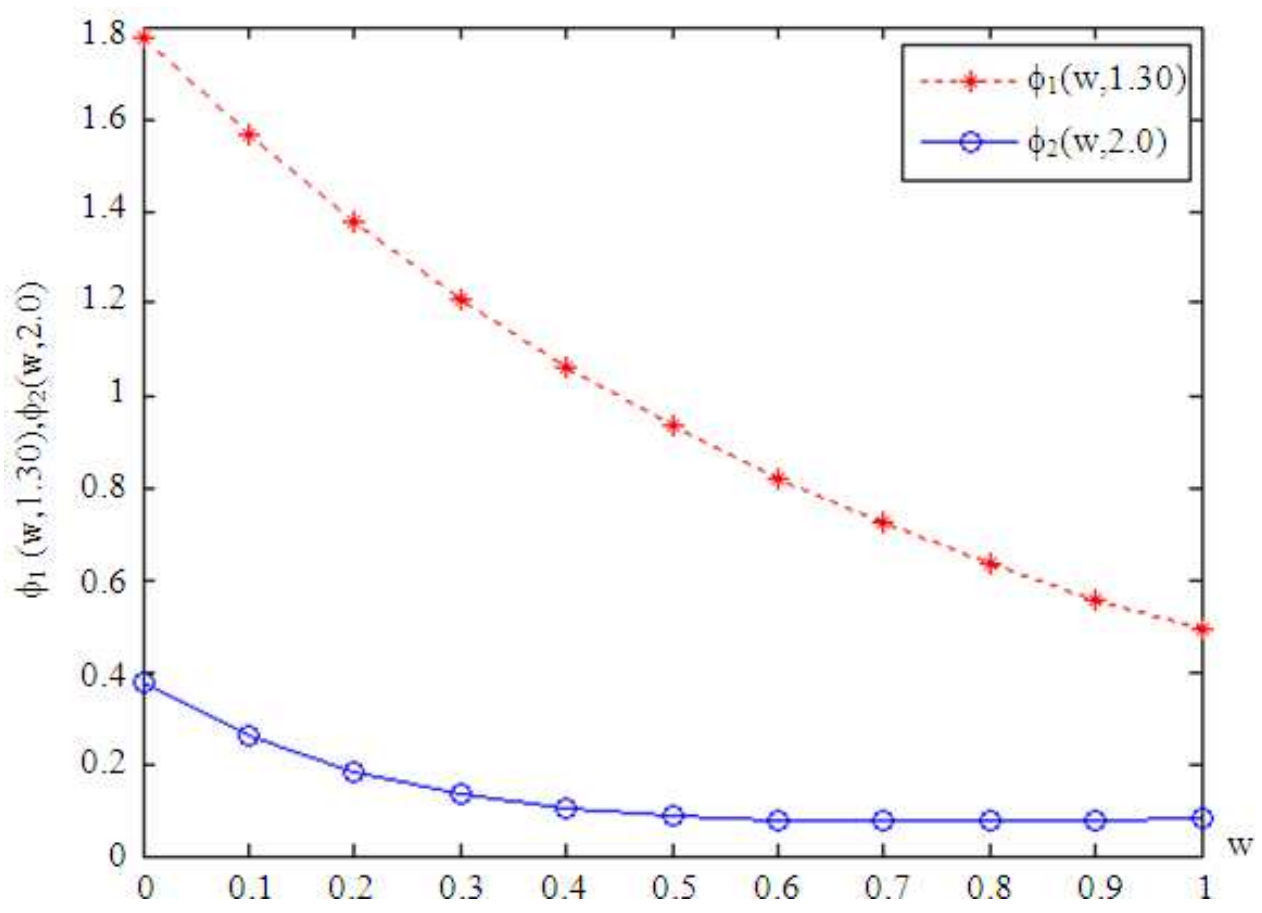

Fig. 1. The graphs of $\phi_{1}(w, 1.30), \phi_{2}(w, 1.95), 0 \leq w \leq 1$, when $\gamma_{1}>\lambda_{1}: \gamma_{1}=1, \gamma_{1}=0.5 ; \gamma_{2}>\lambda_{2}: \gamma_{2}=1.5, \lambda_{2}=1$

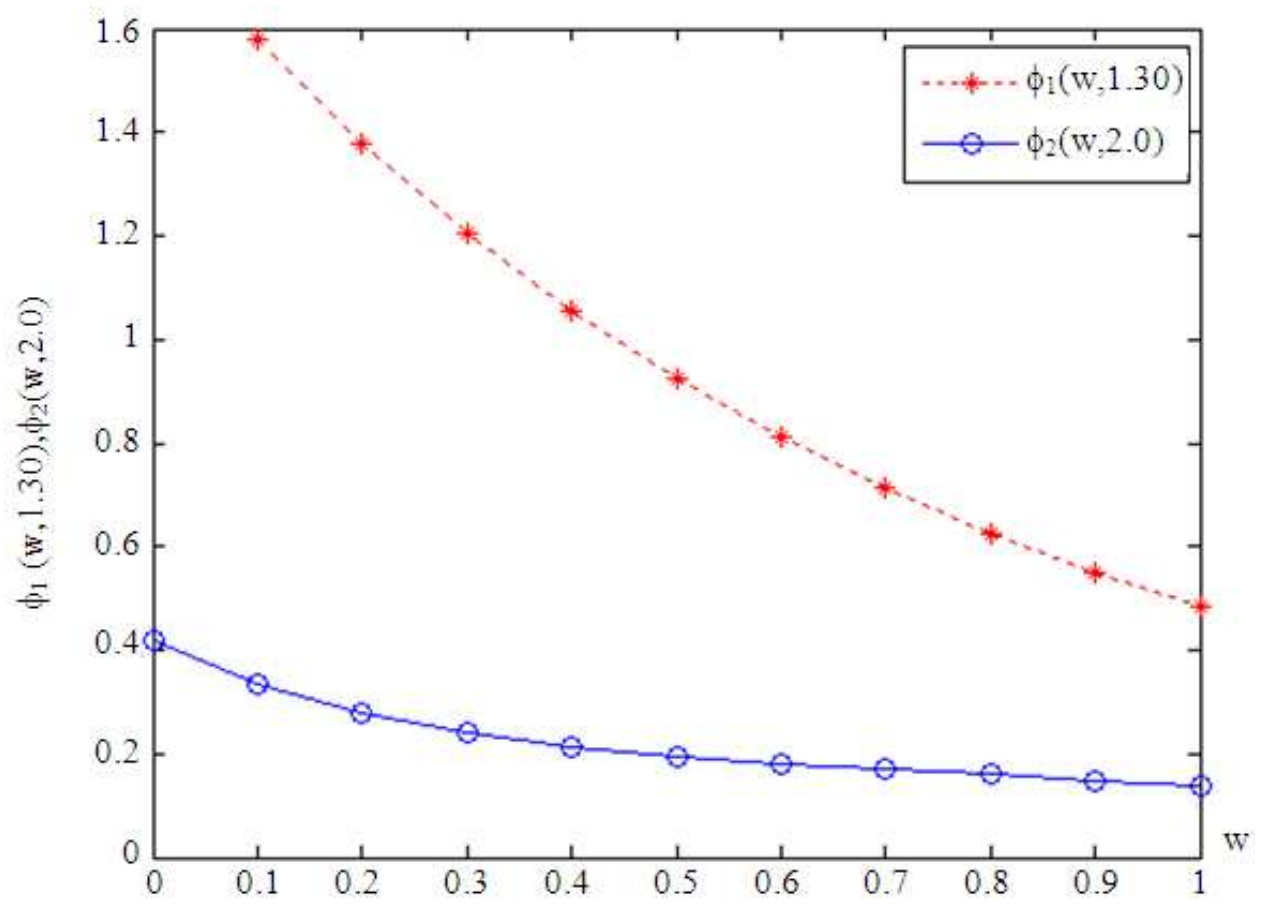

Fig. 2. The graphs of $\phi_{1}(w, 1.30), \phi_{2}(w, 2.0), 0 \leq w \leq 1$, when $\gamma_{1}<\lambda_{1}: \gamma_{1}=0.5, \gamma_{1}=1 ; \gamma_{2}<\lambda_{2}: \gamma_{2}=1, \lambda_{2}=1.5$ 


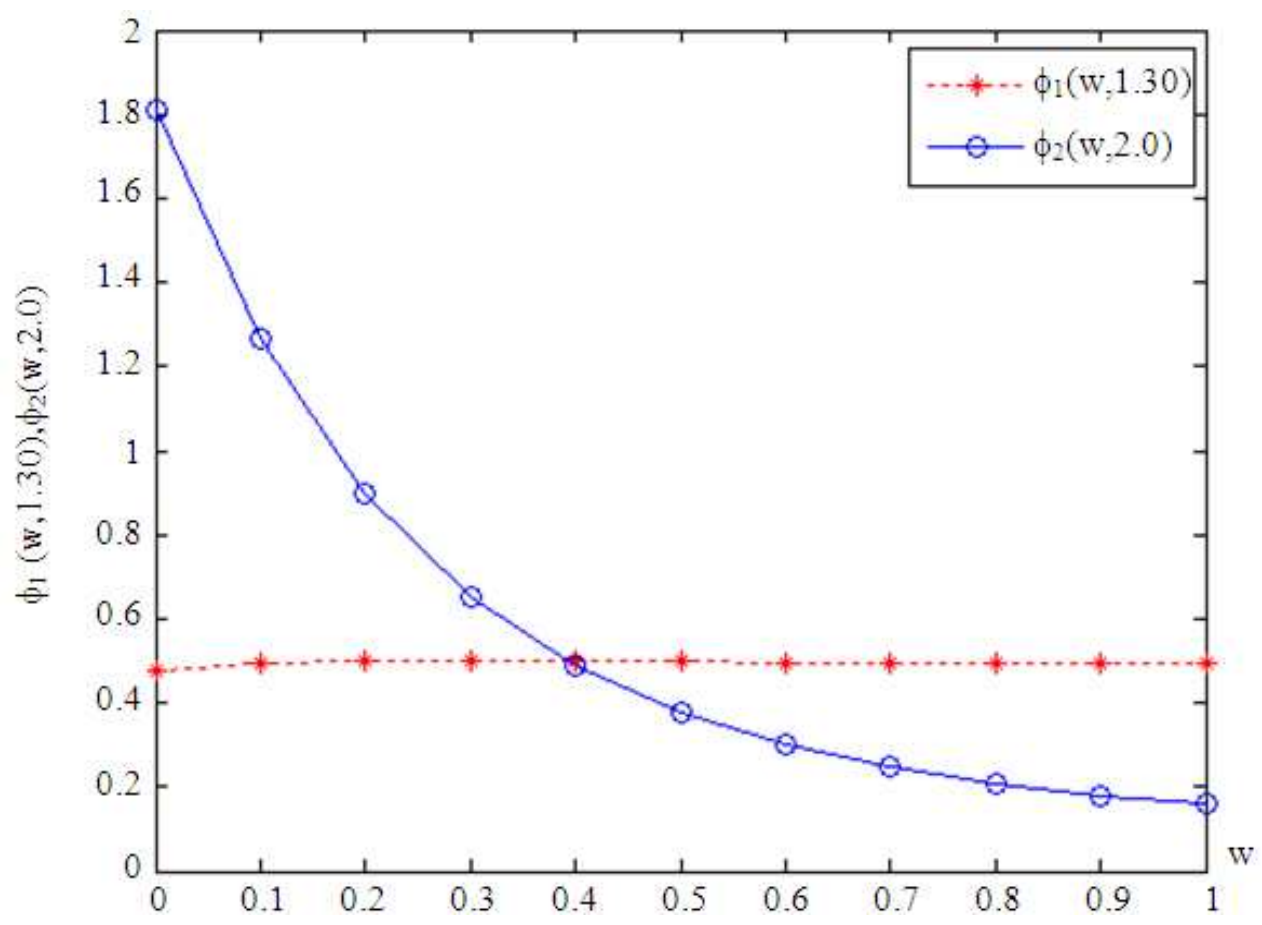

Fig. 3. The graphs of $\phi_{1}(w, 1.30), \phi_{2}(w, 2.0), 0 \leq w \leq 1$, when $\gamma_{1}=\lambda_{1}=\gamma_{2}=\lambda_{2}=1$

So, by Table 4 and Equation (20) either when $\phi_{1}(\mathrm{w}$, $\mathrm{t}), \mathrm{t}=1.30$ or $\phi_{2}(\mathrm{w}, \mathrm{t}), \mathrm{t}=1.95,2.0$, the s.d.f's of them with respect to the three cases (Remark 2.1) can respectively be written as follows:

- $\gamma_{1}>\lambda_{1}: \gamma_{1}=1, \lambda_{1}=0.5 ; \gamma_{2}>\lambda_{2}: \gamma_{2}=1.5, \lambda_{2}=1$ Equation 21a and $21 \mathrm{~b}$ :

$$
\begin{aligned}
& f\left(\phi_{1}, \theta\right)=\frac{0.07683}{\pi} \cdot \frac{\sin ((\mathrm{s}-1.30) \theta)}{\theta}, 0<\theta \leq 2 \pi \\
& \mathrm{f}\left(\phi_{2}, \theta\right)=\frac{0.33313}{\pi} \cdot \frac{\sin ((\mathrm{s}-1.95) \theta)}{\theta}, 0<\theta \leq 2 \pi
\end{aligned}
$$

- $\gamma_{1}<\lambda_{1}: \gamma_{1}=0.5, \lambda_{1}=1 ; \gamma_{2}<\lambda_{2}: \gamma_{2}=1, \lambda_{2}=1.5$ Equation $21 \mathrm{c}$ and $21 \mathrm{~d}$ :

$$
\begin{aligned}
& \mathrm{f}\left(\phi_{1}, \theta\right)=\frac{0.07696}{\pi} \cdot \frac{\sin ((\mathrm{s}-1.30) \theta)}{\theta}, 0<\theta \leq 2 \pi \\
& \mathrm{f}\left(\phi_{2}, \theta\right)=\frac{0.28466}{\pi} \cdot \frac{\sin ((\mathrm{s}-2.0) \theta)}{\theta}, 0<\theta \leq 2 \pi \\
& \text { - } \gamma_{1}=\lambda_{1}=\gamma_{2}=\lambda_{2}=1 \text { Equation } 21 \mathrm{e} \text { and } 21 \mathrm{f}:
\end{aligned}
$$$$
f\left(\phi_{1}, \theta\right)=\frac{0.07686}{\pi} \cdot \frac{\sin ((s-1.30) \theta)}{\theta}, 0<\theta \leq 2 \pi
$$

$$
\mathrm{f}\left(\phi_{2}, \theta\right)=\frac{0.31061}{\pi} \cdot \frac{\sin ((\mathrm{s}-2.0) \theta)}{\theta}, 0<\theta \leq 2 \pi
$$

Figure 4-6 represent the graphs of three pairs ( $\mathrm{f}$ $\left(\phi_{1}, \theta\right)$, f $\left.\left(\phi_{2}, \theta\right)\right)$ corresponding to the three cases (Remark 2.1) and $s=10$ just be chosen to complete the figures.

\subsection{Correlation Coefficients}

By the known Peason's correlation coefficient formula for dependent random variables $\mathrm{X}, \mathrm{Y}$ :

$$
\rho_{X, Y}=\frac{E(X, Y)-E(X) E(Y)}{\sqrt{\operatorname{var}(X) \operatorname{var}(Y)}}
$$

and since each pair of any two p.d.f's $\phi_{1}(w, t)$, $\phi_{2}(\mathrm{w}, \mathrm{t})((18 . \mathrm{a})$ to $(18 . \mathrm{f}))$ with respect to the three cases (Remark 2.1) are dependent. By writing:

$\mathrm{E}(\mathrm{X}, \mathrm{Y})=$ Expectation of the product of $\phi_{1}(\mathrm{w}, \mathrm{t})$ by $\phi_{2}(\mathrm{w}, \mathrm{t})$

$\mathrm{E}(\mathrm{X}) \mathrm{E}(\mathrm{Y})=$ Product of expectation of $\phi_{1}(\mathrm{w}, \mathrm{t})$ by $\phi_{2}(\mathrm{w}, \mathrm{t})$

$\operatorname{Var}(\mathrm{X})=$ Maximum variance of $\phi_{1}(\mathrm{w}, \mathrm{t})$

$\operatorname{Var}(\mathrm{Y})=$ Maximum variance of $\phi_{2}(\mathrm{w}, \mathrm{t})$ 
Mohammad Wahdan Muflih / Journal of Mathematics and Statistics 9 (2): 91-101, 2013

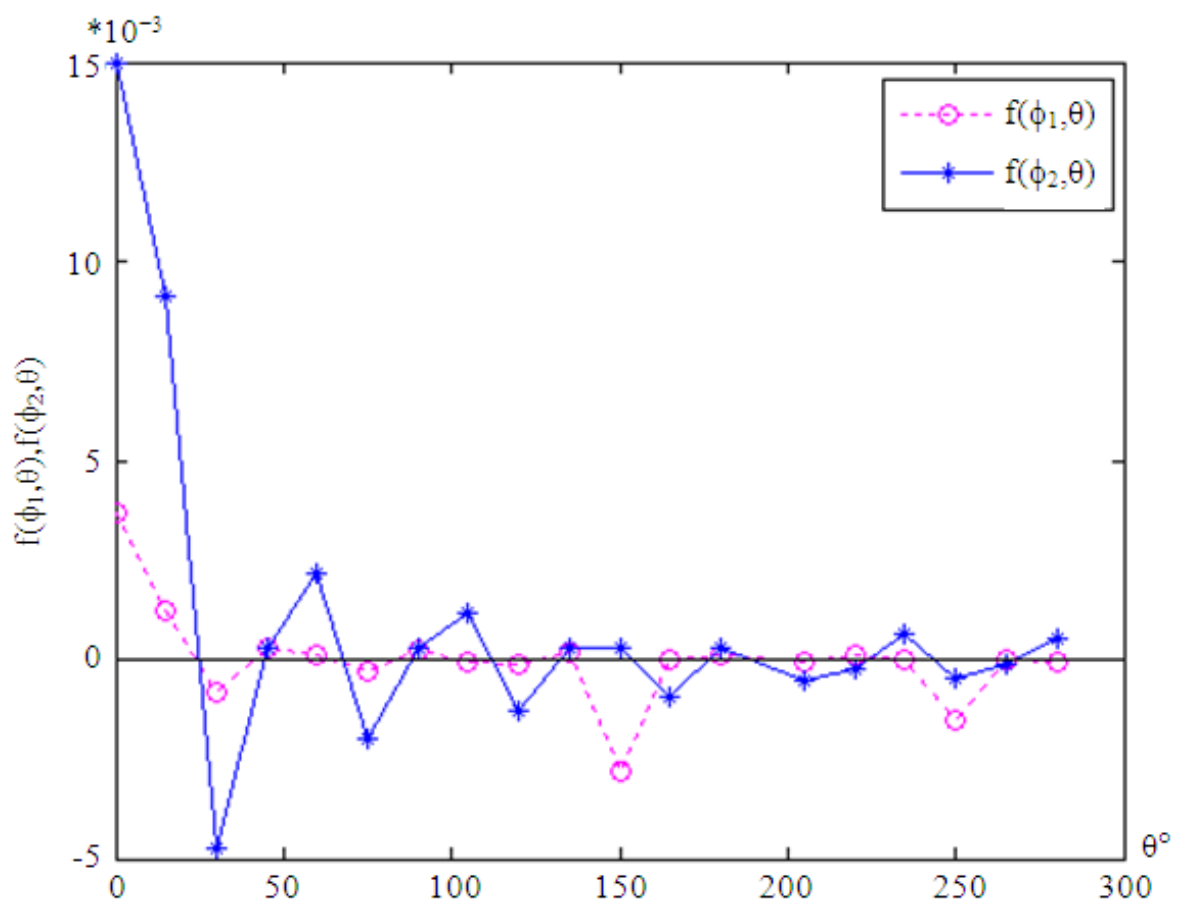

Fig. 4. The graphs of $\mathrm{f}\left(\phi_{1}, \theta\right), \mathrm{f}\left(\phi_{2}, \theta\right), 0 \leq \Theta \leq 2 \pi, s=10$ when $\gamma_{1}>\lambda_{1}: \gamma_{1}=1, \lambda_{1}=0.5 ; \gamma_{2}>\lambda_{2}: \gamma_{2}=1.5, \lambda_{2}=1$

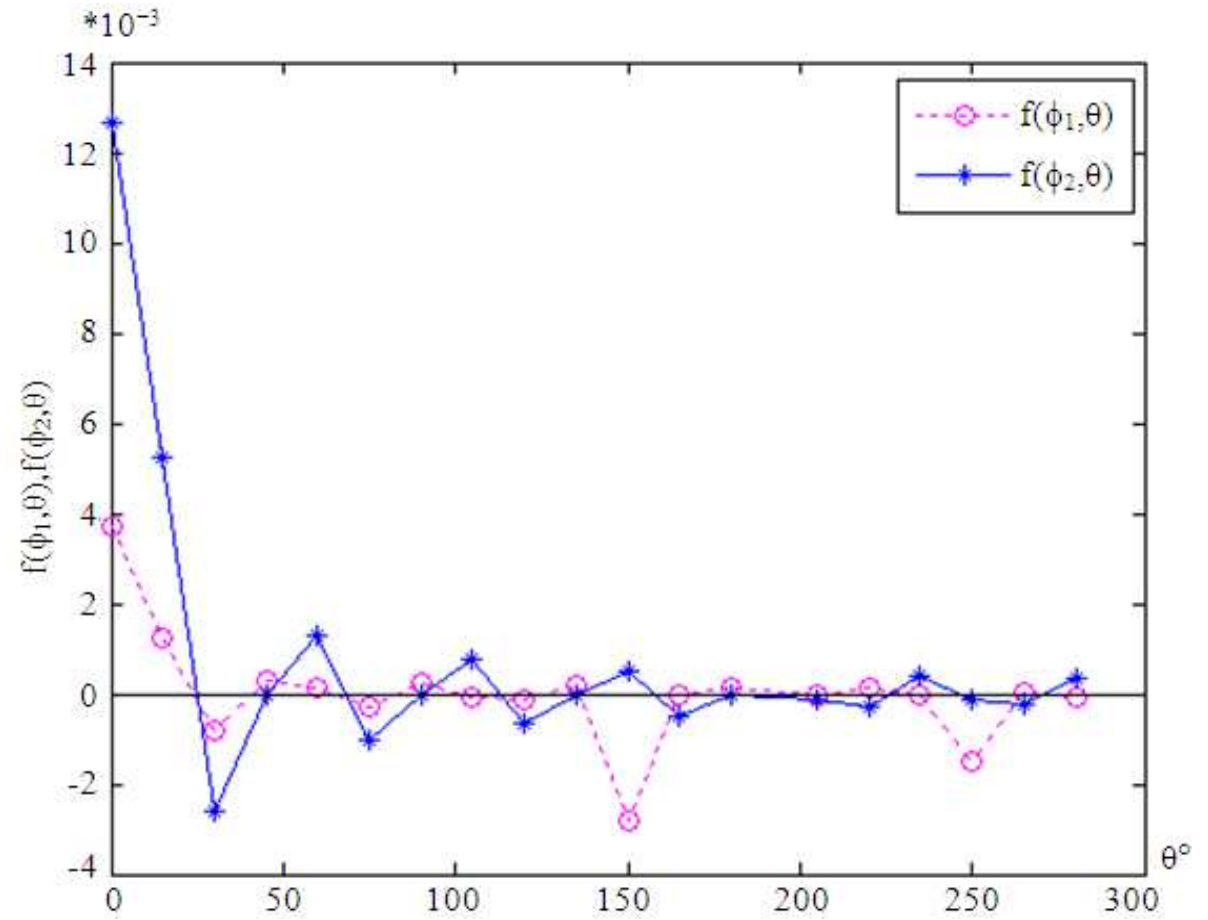

Fig. 5. The graphs of $\mathrm{f}\left(\phi_{1}, \theta\right), \mathrm{f}\left(\phi_{2}, \theta\right), 0<\Theta \leq 2 \pi, s=10$ when $\gamma_{1}<\lambda_{1}: \gamma_{1}=0.5, \lambda_{1}=1 ; \gamma_{2}<\lambda_{2}: \gamma_{2}=1, \lambda_{2}=1.5$ 


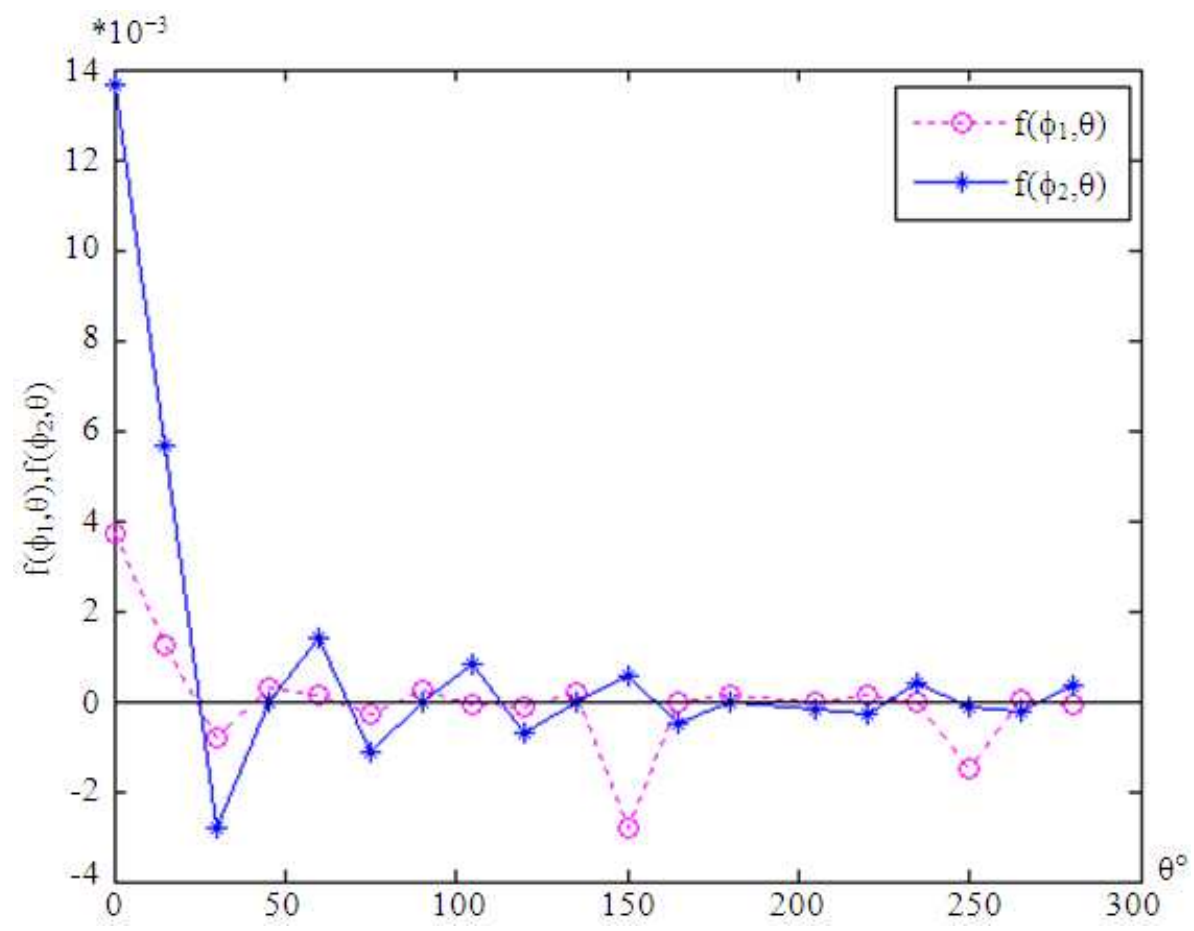

Fig. 6. The graphs of $\mathrm{f}\left(\phi_{1}, \theta\right), \mathrm{f}\left(\phi_{2}, \theta\right), 0<\Theta \leq 2 \pi, s=10$ when $\gamma_{1}=\lambda_{1}=\gamma_{2}=\lambda_{2}=1$

Then:

- $\gamma_{1}>\lambda_{1}: \gamma_{1}=1, \lambda_{1}=0.5 ; \gamma_{2}>\lambda_{2}: \gamma_{2}=1.5, \lambda_{2}=1$ Equation 22a:

$\rho_{\phi_{1}, \phi_{2}}=\frac{0.18077-(0.39535)(0.25522)}{\sqrt{(0.07683)(0.33313)}}=0.49256$

- $\gamma_{1}<\lambda_{1}: \gamma_{1}=0.5, \lambda_{1}=1 ; \gamma_{2}<\lambda_{2}: \gamma_{2}=1, \lambda_{2}=1.5$ Equation $22 \mathrm{~b}$

$\rho_{\phi_{1}, \phi_{2}}=\frac{0.0763-(0.39202)(0.2386)}{\sqrt{(0.07696)(0.28466)}}=0.45233$

- $\gamma_{1}=\lambda_{1}=\gamma_{2}=\lambda_{2}=1$ Equation 22c

$\rho_{\phi_{1}, \phi_{2}}=\frac{0.16964-(0.39514)(0.16725)}{\sqrt{(0.07689)(0.31061)}}=0.67005$

\section{CONCLUSION}

With respect to the three considering cases, the variances of the resulting p.d.f's are slowly decreasing to tending to zero when the times $\mathrm{t}$ is relatively large.
Furthermore, the calculations of the correlation coefficients between any two p.d.f 's having different maximum variances with different times indicate that the best of the three cases is when the parameters $(\gamma, \lambda)$ in both gamma processes are same and equal to one.

As a recommendation, it is possible to use the numerically or analytically solutions for any kinds of integral equations to study some statistical properties of those solutions that is firstly by deriving the p.d.f's. For that, we suggest to consider other cases of the values of the parameters of the gamma processes differ by the cases which studied in this article.

\section{REFERENCES}

AL-Sadany, S.M., 2008. Some Modified Quadrature Methods for Solving Systems of Voltera Linear Integral Equations. M.Sc. Thesis, College of Education, Ibn Al-Haitham, Baghdad University.

Balachandran, K., K. Sumathy and H.H. Kuo, 2005. Existence of solutions of general nonlinear stochastic volterra fredholm integral equations. Stochastic Anal. Applic., 23: 827-851. DOI: $10.1081 /$ SAP-200064487 
Basu, A.K., 2003. An Introduction to Stochastic Processes. 1st Edn., Alpha Science International, Limited, Pangbourne, ISBN-10: 1842651056, pp: 419.

Biazar, J. and A., Rangbar, 2007. A comparison between newton's method and A.D.M for solving special fredholm integral equations. Int. Math. Forum, 2: 215-222.

Emanuel, P., 1962. Stochastic Processes. 1st Edn., SIAM, San Francisco, ISBN-10: 0898714419, pp: 324.

Huda, H.O., 2007. Solutions for the Generalized MultiDimensional Voltera Integral and IntegroDifferential Equations. M.Sc. Thesis, College of Education, Ibn Al-Haitham, Baghdad University.
Milton, J.S., W.J. Padgett and C.P. Tsokos, 1972. On the existence and uniqueness of a random solution to a perturbed random integral equation of the fredholm type. SIAM J. Applied Math., 22: 194-208. DOI: $10.1137 / 0122022$

Vahidi, A.R. and M., Mokhtari, 2008. The Decomposition Method for system of linear fredholm integral equations of the second kind. Applied Math. Sci., 2: 57-62.

Vladimir, K.K. and S. D., Dimitrina, 2009. Gamma process: Pricing path-dependent options. Manage. Sci., 55: 483-496. 